\title{
Determinación de la concentración de solutos en orinas de pacientes caninos: comparación de osmometría versus densidad urinaria (refractometría y tiras reactivas)
}

\author{
Determination of the urine solutes concentration in dogs: osmometry \\ versus urine density (refractometry and reactive strips) compared
}

\author{
Rodríguez JV',2* ,Colla C', Gines MB¹, Schröder Gi \\ 1 Laboratorio Centralizado Hospital Escuela de Grandes y Pequeños Animales, (HEGYPA). \\ Facultad de Ciencias Veterinarias; 2 Centro Binacional de Investigaciones en Criobiología \\ Clínica y Aplicada (CAIC). Universidad Nacional de Rosario (UNR). \\ * Correo electrónico del autor de contacto:jrodrig@fbioyf.unr.edu.ar
}

\begin{abstract}
Resumen
Para estimar la concentración de solutos urinarios, los métodos disponibles son la osmometría (OSM) (método de referencia) y la densidad urinaria (DU) que puede ser determinada mediante la densimetría, la refractometría (RF) y las tiras reactivas (TR). En veterinaria se usa tradicionalmente la RF, aunque recientemente se ha detectado la tendencia en algunos laboratorios al uso de TR. En la literatura existen controversias acerca de la eficacia de la TR respecto de su uso en muestras de caninos y felinos. Los objetivos de este estudio fueron: valorar la exactitud de la RF y de las TR en la determinación de la DU en la orina de caninos comparándolas con el método de referencia, la OSM, y establecer un criterio para la valoración de la DU en las muestras de orina recibidas en nuestro laboratorio. Se analizaron 35 muestras de pacientes caninos. Se compararon: 1-osmolalidad de las muestras mediante la técnica de descenso crioscópico, 2- DU utilizando un refractómetro clínico y 3- DU utilizando tiras reactivas comerciales. Los resultados mostraron una fuerte correlación entre los valores de la DU determinados por RF y la OSM con una clara tendencia lineal. En cambio, la correlación entre la DU estimada por TR y la OSM fue muy débil, mostrando la falta de asociación entre dichas mediciones. Estos resultados sugieren que las TR muestran un valor posible de la DU, pero no su valor real. Para estimar la DU en caninos, la RF es el método óptimo en términos de costos, medición en campo, simpleza y fiabilidad.
\end{abstract}

\section{Palabras clave}

Osmometría, refractometría, tiras reactivas, orina, caninos

\begin{abstract}
Several methods are available for urinary solute concentration determination, such as osmometry (OSM) (reference method) and urine density (UD). UD can be determined by urinometry, refractometry (RF) and reactive strips (RS). In veterinary medicine, RF is the most commonly used method; however, the use of RS to determine UD is increasing in many diagnostic laboratories. The objectives of this investigation were to evaluate the accuracy of RF and RS on the UD determination of canine urine compared to the reference method, and to establish an analytical criterion for the DU estimation of the urine samples received in our laboratory. In this study, 35 urine samples from canine patients were analyzed. We compared the results obtained using three analytical methods: 1- Osmolality by the freezing point depression method, 2- UD by a clinical refractometer and 3- UD using commercial RS. The readings from the clinical refractometer showed an excellent correlation with the osmolality results, with a lineal tendency between both parameters. However, the correlation between UD estimated by RS and osmolality was very weak, showing lack of association between both methods. These results suggest that the RS could provide an approximate but not accurate UD value, and that RF is a more appropriate method in terms of cost, use in the field and accuracy for the assessment of UD in dogs.
\end{abstract}

\section{Key words}

Osmometry, refractometry, reactive strips, urine, canine

Fecha de recepción: 12/10/2017

ANALECTA VeT 2018; 38(1): 45-49

Fecha de aprobación: 02/03/2018

Impresa ISSN 03655 14-8 Electrónica ISSN 1514-2590

doi.org/10.24215/15142590e024 


\section{Introducción}

Para estimar la concentración de solutos en la orina existen dos métodos, 1- la osmometría (OSM), considerada la técnica de referencia, (Watson, 1998) y 2- la determinación de la densidad urinaria (DU), equivalente al peso específico en el sistema métrico decimal. Para determinar la DU pueden utilizarse técnicas como la refractometría (RF), la urinometría (densímetro) y las tiras reactivas (TR). En el laboratorio clínico veterinario, debido al pequeño volumen de las muestras de orina, se utiliza tradicionalmente la RF, aunque recientemente se ha detectado la tendencia en algunos laboratorios al uso de TR, tanto manual como automatizado con lectores de TR. En la literatura existen controversias acerca de la eficacia de la TR (diseñada para orina humana) respecto de su uso en caninos y felinos (Dossin et al., 2003; Sink \& Weinstein, 2012). Dada la importancia creciente de la aplicación de las Buenas Prácticas de Laboratorio (BPL) en los laboratorios clínicos veterinarios es imprescindible estandarizar protocolos con referencia a la metodología a aplicar en el análisis de las muestras. Los objetivos de este estudio fueron: a- valorar la exactitud de la RF y de las TR en la determinación de la DU en la orina de caninos comparándolas con el método de referencia (OSM) y b- establecer un criterio para la valoración de la DU en las muestras de orina recibidas en nuestro laboratorio.

\section{Descripción metodológica}

Se analizaron 35 muestras de orina provenientes de un universo de pacientes caninos de distintas edades, sexo y razas. Las muestras fueron recibidas y procesadas en el laboratorio, se excluyeron las muestras que contenían glucosa y, en los casos en que se detectaron trazas de proteínas, se aplicó el criterio que se detalla en el texto. La presencia de glucosa y proteínas se estimó mediante la TR (Siemens Multistix 1056, Alemania). En todas las muestras se determinó la concentración de proteínas urinarias utilizando la técnica espectrofotométrica del rojo de pirogalol (Proti U/LCR, Wiener Lab, Argentina) y la concentración de creatinina urinaria mediante la reacción de Jaffé (Biggs \& Cooper, 1961). El criterio para la exclusión de muestras con presencia de proteínas fue: concentración de proteínas urinarias $>270 \mathrm{mg} / \mathrm{l}$ y relación proteína/ creatinina $>0,20$ (Tvedten, 2016). Para valorar la exactitud de la RF y de las TR se decidió comparar los resultados obtenidos utilizando los tres métodos: 1- determinación de la osmolalidad de las muestras mediante la técnica de descenso crioscópico (Osmomat 030, Gonotec, Alemania, calibración del instrumento mediante estándares de 300,850 y $2000 \mathrm{mOsm} / \mathrm{KgH}_{2} \mathrm{O}$ ), 2- determinación de la DU utilizando un refractómetro clínico (Alla France mod. 95000-017, Francia) y $3^{-}$ determinación de la DU utilizando tiras reactivas (Siemens Multistix 1056, Alemania). Esta última fue realizada por un sólo operador por comparación colorimétrica visual con la escala provista en el kit.

En las muestras se determinó la DU por RF y TR y luego fueron conservadas a $-20{ }^{\circ} \mathrm{C}$ hasta la determinación de la OSM. En los casos en que la DU excedió los límites establecidos en el refractómetro (DU >1,050) y en TR (DU >1,030) las muestras fueron diluidas $1 / 2$ con agua destilada y luego analizadas. Para determinar la DU de la dilución se multiplicó la porción decimal de la nueva lectura por la dilución realizada (por ejemplo: DU determinada en dilución $1 / 2=1,020$, luego: $0,020 \times 2=0,040$, entonces la DU corregida será 1,040) (Sink \& Weinstein, 2012).

\section{Discusión y conclusiones}

Los resultados mostraron una fuerte correlación entre los valores de la DU determinados por RF y la OSM, $(r=0,9884)$, con una clara tendencia lineal (Fig. 1A). También ello sucedió para los valores de DU >1,050 que fueron diluidos. En cambio, la correlación entre la DU estimada por TR y la OSM fue muy débil $(r=0,4306)$, mostrando una falta de asociación entre dichas mediciones (Fig. 1B). Debido a la dispersión de los datos obtenidos no podemos determinar si la interferencia presentada en el análisis por TR sobre o subestima la DU.

Cuando se correlacionaron los valores de DU determinados por RF y TR también se encontró una asociación débil entre ellos (DU-RF, $\mathrm{r}=0,3844$; DU-TR $=0,668+0,342, \mathrm{n}=35$ ) (Fig. $1 \mathrm{C})$.

Respecto de la concentración urinaria de glucosa, proteínas y creatinina, ninguna muestra contenía glucosa. La relación proteína/creatinina en la orina se utilizó como criterio para compensar la dilución o concentración de la orina en la excreción de analitos como las proteínas (Trumel et al., 2004). En este trabajo, las concentraciones de proteínas oscilaron entre 14-268 mg/l (mediana $192 \mathrm{mg} / \mathrm{l}$ ), las concentraciones de creatinina urinaria fueron de 93-2940 mg/l (mediana 1131 $\mathrm{mg} / \mathrm{l}$ ) y las relaciones $\mathrm{P} / \mathrm{C}$ fueron de $0,01-0,203$ (mediana 0,074 ) para las 35 muestras de orina analizadas.

La TR estima indirectamente la DU utilizando la medida de la fuerza iónica de la orina. El principio básico de este método considera que los constituyentes iónicos y no iónicos de la orina están presentes en proporción constante y esto no es siempre así (Chadha et al., 2001). Esta determinación es afectada por la composición iónica de la orina, las proteínas que tienen carga eléctrica en solución y por el pH de la misma, mayormente en el rango alcalino (Dorner et al., 1984). Por estas razones, solamente las orinas con un $\mathrm{pH}$ de 7 a 7,5 deberían ser analizadas utilizando 
TR; en nuestro caso el rango de $\mathrm{pH}$ de las orinas analizadas fue de 6,0 a 8,5. Además, para la misma concentración iónica total, diferentes iones afectan de distinta manera la DU, por ejemplo: para orinas con osmolalidad constante los cationes como el $\mathrm{NH}^{4+}, \mathrm{Ca}^{2+}$ y $\mathrm{Mg}^{2+}$ incrementan la DU desproporcionadamente con respecto al $\mathrm{Na}^{+}$y al $\mathrm{K}^{+}$ (Dorner et al., 1984). En cambio, la glucosuria o proteinuria incrementan las lecturas refractométricas de la orina; por ello, es importante realizar las correcciones apropiadas en la DU (Chadha et al., 2001). Para las determinaciones de DU diarias utilizamos los factores de corrección informados en la bibliografía (Pradella et al., 1988).

Los resultados obtenidos indican claramente que las TR no deben utilizarse para estimar la DU en caninos, siendo la RF el método óptimo en términos de costos, medición en campo y fiabilidad.

Como consecuencia de la investigación realizada, se estableció entonces la siguiente secuencia para la determinación de la DU en el análisis completo de la orina realizado en muestras de caninos (Fig. 2):

1- análisis con TR, 2- centrifugación, 3- análisis de la muestra por RF, si el valor obtenido es $>1,050$ se diluye la muestra $1 / 2$ con agua destilada y se corrige el resultado, 4- nueva determinación de la $\mathrm{RF}$, realizando el cálculo correspondiente, $5^{-}$en caso de detectar glucosa o proteínas, determinación de las concentraciones de las mismas utilizando las técnicas analíticas correspondientes y realización de las correcciones respectivas a la DU determinada por RF. Por cada g/l de proteína urinaria, se sustraen 0,003 unidades a la DU medida (su impacto sobre la osmolalidad es de aproximadamente $3 \mathrm{mOsm} / \mathrm{kg} \mathrm{H}_{2} \mathrm{O}$ ), en cambio, para glucosa, por cada $10 \mathrm{~g} / \mathrm{l}$ de glucosa la Osm se incrementa en $55 \mathrm{mOsm}(180 \mathrm{~g} / \mathrm{l}$ de glucosa equivalen a $1000 \mathrm{mOsm}$ ), por ello el factor de corrección es la sustracción de o,004 unidades a la DU determinada (Pradella et al., 1988).

Hemos sugerido realizar el análisis de orina con TR en primera instancia debido a que de esta manera podemos establecer qué orinas contienen glucosa o concentraciones elevadas de proteínas y así organizar el análisis fisicoquímico de las orinas en dos grupos diferentes: las normales, que son procesadas inmediatamente, y las presuntamente patológicas, que requieren determinación de la concentración de glucosa, proteínas, etc., por otros métodos analíticos.

\section{Agradecimientos}

Agradecemos a Wiener Lab, por la generosa donación del kit Proti U/LCR. Los equipos utilizados en este trabajo fueron provistos por el subsidio del Servicio de Relaciones Internacionales y Partenariado Territorial de la Región Friuli-Venezia-Giulia de Italia para la ejecución del proyecto "Creación del Centro Binacional (Argentina - Italia) de Investigación en Criobiología Clínica y Aplicada“. Protocollo: n. 19096/PT.
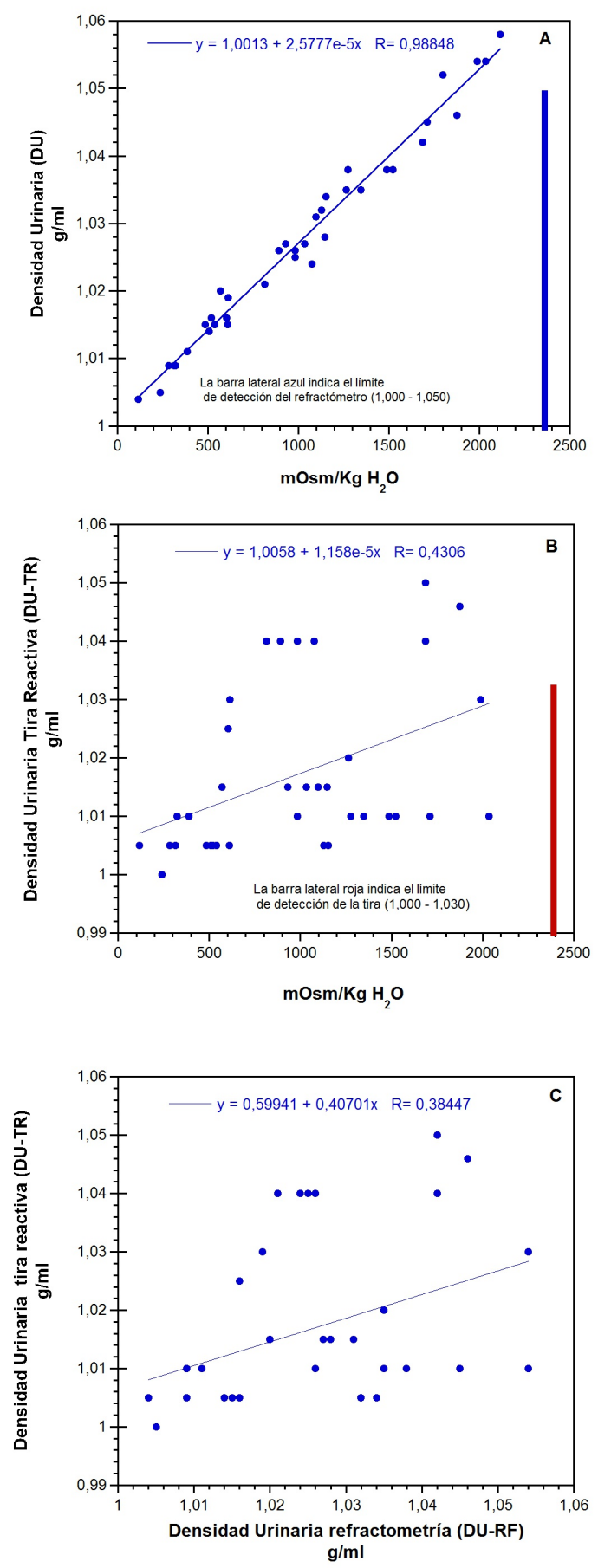

Figura 1. A. Relación entre la densidad de la orina medida por refractometría (DU-RF) y la osmolalidad de la orina (mOsm/ $\mathrm{Kg} \mathrm{H} 2 \mathrm{O}$ ), $\mathrm{n}=35$ muestras. B. Relación entre la densidad de la orina medida por tiras reactivas (DU-TR) y la osmolalidad de la orina ( $\mathrm{mOsm} / \mathrm{Kg} \mathrm{H} 2 \mathrm{O}), \mathrm{n}=35$ muestras. C. Relación entre la densidad de la orina analizada mediante refractometría (DURF) con la densidad de la orina determinada mediante tiras reactivas (DU-TR), $\mathrm{n}=35$ muestras. 


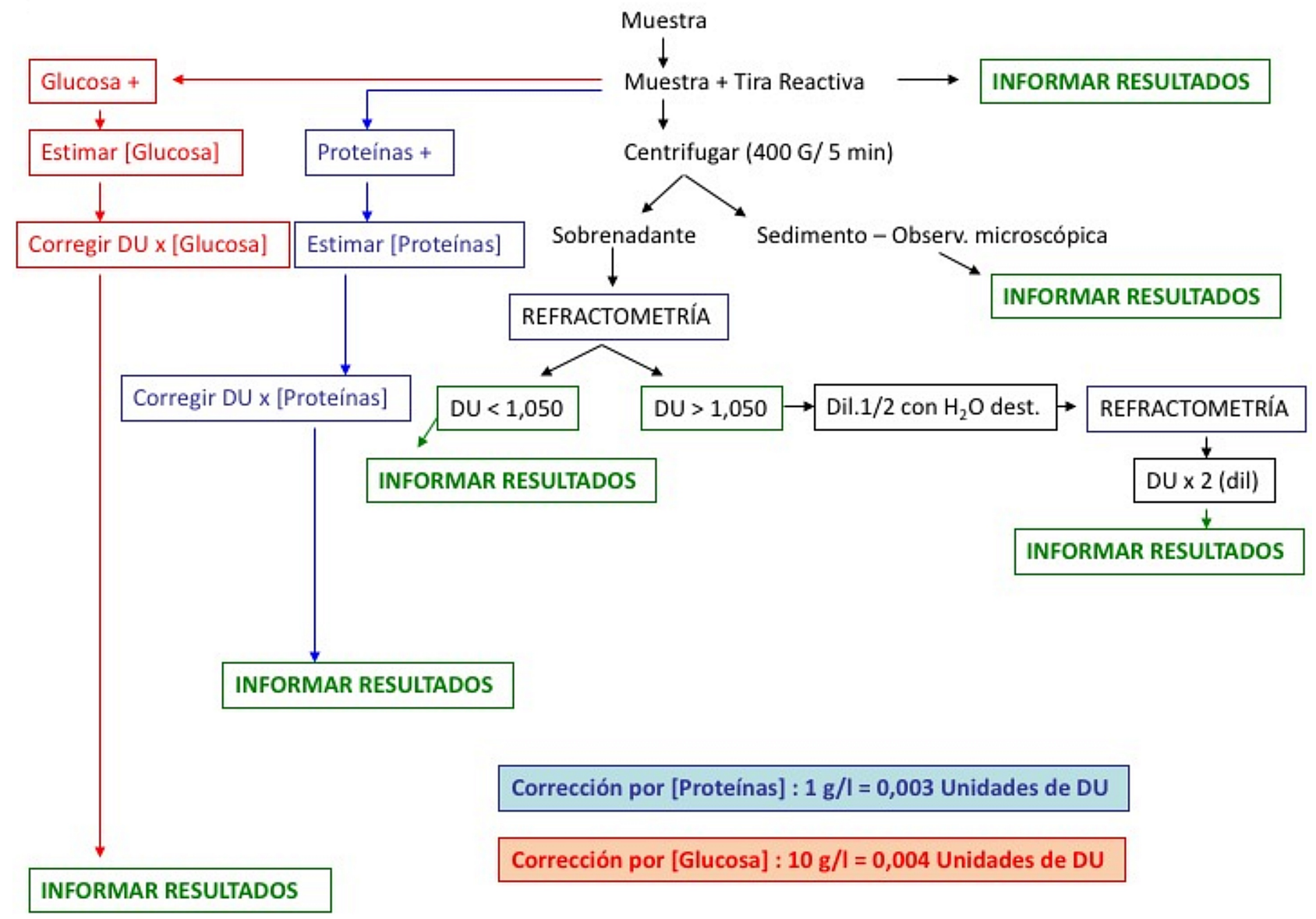

Figura 2. Esquema propuesto para el análisis completo de orina en muestras de caninos.

\section{Conflicto de intereses}

Todos los autores declaran que no existe conflicto de intereses, relaciones financieras, personales o de otro tipo con personas $u$ organizaciones que pudieran afectar al presente trabajo.

\section{Bibliografía}

Biggs HG, Cooper JM. 1961. Modified Folin methods for the measurement of urinary creatine and creatinine. Clinical Chemistry 7(6):655-64.

Chadha V, Garg U, Alon US. 2001. Measurement of urinary concentration: a critical appraisal of methodologies. Pediatric Nephrology 16(4):37482.

doi:10.1007/s004670000551

Dorner K, Campos R, Bornser S. 1984. Further evaluation of the SG test strip for estimation of urinary osmolality. Journal of Clinical Chemistry and Clinical Biochemistry 22(6):419-25.

Dossin O, Germain C, Braun J. 2003. Comparison of the techniques of evaluation of urine dilution/ concentration in the dog. Journal of Veterinary Medicine Series A Physiology Pathology Clinical Medicine 50(6):322-5.

doi: 10.1046/j.1439-0442.2003.00542.x

Pradella M, Dorizzi RM, Rigolin F. 1988. Relative density of urine: methods and clinical significance. CRC Critical Reviews in Clinical Laboratory Sciences 26(3):195-242.

doi:10.3109/10408368809105890

Sink CA, Weinstein NM. Chapter 3: Routine Urianalysis: Physical properties. En: Urine Practical Veterinary Urinalysis. 2012. West Sussex, UK: Wiley-Blackwell. pp. 23-24.

Tdveten H. 2016. Urine protein total concentration in clinically normal dogs. Veterinary Clinical Pathology 45(3):395-6. doi: 10.1111/vcp.12379 
Trumel C, Diquélou A, Lefebvre H, Braun JP. 2004. Inaccuracy of routine creatinine measurement in canine urine. Veterinary Clinical Pathology 33(3):128-32.

doi: 10.1111/j.1939-165X.2004.tboo361.x

Watson ADJ. 1998. Urine specific gravity in practice. Australian Veterinary Journal 76(6):3928.

doi: 10.1111/j.1751-0813.1998.tb12384.x 